\title{
Factors Influencing Lebanese Medical Students' Decisions to Train Abroad: Evaluation of a Conceptual Framework
}

\author{
Elie A. Akl*,1,2,3, Nancy Maroun ${ }^{4}$, Carl K. Li $^{3}$, Brydon J.B. Grant ${ }^{1,3}$ and Holger J. Schünemann ${ }^{2,5}$ \\ ${ }^{1}$ Department of Medicine, State University of New York at Buffalo, NY, USA \\ ${ }^{2}$ Department of Clinical Epidemiology and Biostatistics, McMaster University, Hamilton, ON, Canada \\ ${ }^{3}$ Department of Social and Preventive Medicine, State University of New York at Buffalo, NY, USA \\ ${ }^{4}$ Department of Sociology, State University of New York at Buffalo, NY, USA \\ ${ }^{5}$ Department of Medicine, McMaster University, Hamilton, ON, Canada
}

\begin{abstract}
Objective: To evaluate a conceptual framework portraying the factors underlying the intentions of medical students to train abroad.

Methods: Eligible subjects were students in the pre-final and final years of medical studies in Lebanon. A survey questionnaire based on the conceptual framework inquired about demographic and educational characteristics, influencing factors, and intentions to train abroad. We assessed each of the factors for its frequency, distribution, and association with the intention to train abroad.

Results: Of 576 eligible students, 425 (74\%) participated. The frequency of the framework factors varied from $16 \%$ to $94 \%$ and was the highest for factors related to training and professional career. The factors independently associated with the intention to train abroad related to residency training, concern with professional career and political conditions. The intention to train abroad was also associated with thinking that the general public considers doctors trained abroad as more qualified (OR=6.3; 95\% $\mathrm{CI}=1.2-33.2$ ); receiving information about abroad training from doctors trained or training abroad $(\mathrm{OR}=9.7$; 95\% $\mathrm{CI}=1.7-56.6)$; having relatives or friends living abroad $(\mathrm{OR}=6.9$; 95\% CI=1.4-35.7); and witnessing residents traveling to retrain abroad (OR=22.2; 95\% $\mathrm{CI}=4.5-111.1)$.

Conclusions: Issues related to residency training, concerns about future career, and political conditions are the main motivators for Lebanese medical students to train abroad. The proposed conceptual framework provides a useful tool, but requires further evaluation.
\end{abstract}

Keywords: Medical student, medical training, physician migration, Lebanon.

\section{INTRODUCTION}

As demands on health systems have been rapidly rising worldwide, demands on their human resources have also been growing [1]. Many high income countries responded to these demands by recruiting healthcare workers mainly from low income countries (LIC), resulting in a large wave of international migration of physicians [2, 3]. This phenomenon is likely to worsen with the projected physician shortages in high income countries [4-7], and plans to remediate these shortages, at least partly, through more international recruitment [8].

The Lebanese physician workforce has endured severe losses through international migration. Indeed, Lebanon has the highest emigration factor in the Middle East and North Africa and the 7th highest in the World [8]. The United Sates (US) physician workforce, alone, included in 2004 about

*Address correspondence to this author at the Department of Medicine, State University of New York at Buffalo ECMC-CC 142, 462 Grider St. Buffalo, NY 14215, USA; Tel: ++ 1 716-898-5793;

Fax: ++1 716-898-3119; E-mail: elieakl@buffalo.edu
$40 \%$ of Lebanese medical graduates of the preceding 25 years [9]. After adjusting for country population size, Lebanon ranked $2^{\text {nd }}$ as source country of international medical graduates practicing in the US [9]. In 2005, 96\% of students of Lebanese medical schools intended to train abroad [10].

We have previously developed, using qualitative methodology, a conceptual framework depictingthe factors influencing the decisions of graduating Lebanese medical students to train abroad [11]. The main influencing factors were categorized as push, pull, retain and repel factors and related to the following: residency training, professional career, personal conditions, political conditions, and social conditions. There were three additional factors: societal expectations that students should train abroad; marketing of abroad training; and an established culture of migration [11]. There were also perceived "barriers" to abroad training. However, this proposed framework has not been evaluated.

The objective of the present study was to quantitatively assess the main motivators for Lebanese medical students to train abroad and to evaluate the proposed conceptual frame- 
work by assessing each of its factors for its frequency, distribution, and association with the intention to train abroad.

\section{MATERIALS AND METHODOLOGY}

We used a survey methodology to collect data for this study.Eligible subjects werestudents in the pre-final and final years of medical studiesin Lebanon. We excluded one recently established medicalschool, as it did not have a prefinal or a final year class at the time. The Institutional Review Boards of all involved institutions approved the study (American University of Beirut, Beirut Arab University, Lebanese University, Saint Joseph University, and University of Balamand).

\section{Questionnaire}

The questionnaire was developed using the results of a qualitative study ofthe intentions and motives of Lebanese medical students to train abroad [11]. The participants inthe qualitative study were recruited from the same target population. The qualitative study was conductedabout 4 months prior to this survey. The questionnaire included questions relating to (see Appendix):

(1) Student's demographic characteristics (age, sex, marital status, socio-economic status (SES), having a Lebanese citizenship, and having an additional citizenship) and educational characteristics (medical school, year of medical education, planned residency type).

(2) The main influencing factors: residency training, professional career, personal conditions, political conditions and social conditions. The additional factors were: societal expectations, marketing of abroad training, and the culture of migration.

(3) The perceived barriers to abroad training: expenses associated with training abroad, the required process of certification, getting visas, and other.

The questionnaire included additional questions about student's intention to train abroad, the results of which are reported elsewhere [10]. All questions had a closed ended format. Answer options for most questions consisted of 7point Likert scales ranging from -3 to +3 with the following anchors: "I strongly disagree" (for -3 ), "I neither agree or disagree" (for 0 ) and "I strongly agree" (for +3 ). The questionnaire was in English which as a language of instruction in all medical schools, albeitto varying degrees. In order to ensure the clarity and feasibility of the questionnaire we pilot tested it with a convenience sample of10 physicians who had recently graduated from Lebanese medical schools.

\section{Data Collection}

During the months of October and November 2005, an investigator from each of the five participating medical schools invitedeligible students to participate in the study. These were verbal invitations at the end of a class. Willing participantsfilled out a paper-basedanonymous and confidential survey questionnaire. Eligible students were contactedon a second occasion to invite thosewho had not participated to consider doing so.

\section{Data Analysis}

We first conducted a descriptive analysis of students' demographic and educational characteristics using mean and standard deviation for continuous variables and frequencies and percentages for categorical variables.

For the evaluation of the theoretical framework we conducted for each of its factors the following series of analyses:

1. To assess the frequency of each factor, we conducted a descriptive analysis for each factor using percentages.

2. To assess the distribution of each factor, we conducted a bivariate analysis using Student's t-test and the ChiSquare test to assess whether a factor is associated with any of the demographic or educational variables. We then conducted a multivariable analysis using logistic and multinomial regression models with each factor as the dependent variable and the demographic and educational characteristics as the independent variables.

3. To assess whether intention to train abroad was associated with any of the factors of the conceptual framework, we conducted multivariable analyses using logistic regression modelswith backward eliminationwith the intention to train abroad as the dependent variable(with yes/no categories), the factor as the independent variables and demographic and educational characteristics as the covariates; we considered only the factors that were associated with the intention to train abroad in the bivariate analysis.

Finally, we used the statistical results to modify the above mentioned conceptual framework for medical for medical students' decisions to train abroad [11]. The modification aimed to highlight the most prevalent factors and those that are independently associated with the intention to migrate.

We considered two-sided $\mathrm{p}$ values and $\mathrm{p}<0.05$ as statistically significant. We used Microsoft Office Excel 2003 for data management and SPSS, version 13.0 (SPSS, Inc., Chicago, Illinois), for data analyses. The strengths of the relationships of the variables in the regression analyses are expressed as odds ratios (OR), indicating for example the relativeodds of the tested factor to be associated withintending compared tonot intending to train abroad.

\section{RESULTS}

Of 576 eligible students, 430 responded to the survey. We excluded 5 responses because of missing data. The survey response rate was thus 74\%. The Table 1 shows the demographic and educational characteristics of respondents.

\section{Influencing Factors}

Most students considered the factors related to residency training as motivators to train abroad: residency training opportunities (78\%); clinical training (94\%); research training (85\%); teaching in residency programs (83\%); working conditions of residents (86\%); financial situation of residents (89\%); and impact of residency training on future career (92\%). Similarly, most students considered the factors related to working as a doctor as motivators to train abroad: 
Table 1. Baseline Characteristics of Respondents to a2005 Survey of Medical Students in Lebanon (N=425)

\begin{tabular}{|c|c|c|}
\hline Age (years) & & Mean=23.8; SD=1.4 \\
\hline \multirow[t]{3}{*}{ Marital Status } & Single & $410(96)$ \\
\hline & Married & $6(1)$ \\
\hline & Widow & $0(0)$ \\
\hline \multirow[t]{3}{*}{ Socio-economic status } & Lower & $7(2)$ \\
\hline & Lower middle & $101(24)$ \\
\hline & Upper middle & $238(56)$ \\
\hline Lebanese citizenship & Yes & $401(94)$ \\
\hline Additional citizenship* & Yes & $72(17)$ \\
\hline \multirow[t]{5}{*}{ Medical school } & American University of Beirut & $134(32)$ \\
\hline & Beirut Arab University & $51(12)$ \\
\hline & Lebanese University & $113(27)$ \\
\hline & Université Saint Joseph & $61(14)$ \\
\hline & University of Balamand & $66(16)$ \\
\hline \multirow[t]{2}{*}{ Year of medical school } & Pre-final year & $215(51)$ \\
\hline & Final year & 207 (49) \\
\hline \multirow[t]{4}{*}{ Planned residency type } & None & $6(1)$ \\
\hline & Surgical & $137(32)$ \\
\hline & Medical & $241(57)$ \\
\hline & Other & 36 (9) \\
\hline
\end{tabular}

* Indicates having an additional citizenship in addition to the primary citizenship

job opportunities (92\%); working conditions of doctors (92\%); and financial situation of doctors (92\%). About half the students considered the following factors as motivators to train abroad: personal conditions (46\%); social conditions (52\%); and political conditions (46\%).

The associations between theconsideration of a particular influencing factor as a motivator, and demographic and educational characteristics were not significant in the multivariable analyses. This suggests that none of these characteristics is independently associated withconsidering any of these factors as a motivator.

However, the associations between theintention to train abroad and considering the following influencing factors as motivatorswere statistically significant: clinical training $(\mathrm{OR}=30.7$; 95\% CI=4.5-207.8); working conditions of residents (OR=48.5; 95\% CI=6.2-382.5); financial conditions of residents $(\mathrm{OR}=8.5 ; 95 \% \mathrm{CI}=1.6-45.1)$; impact on career $(\mathrm{OR}=15.9$; 95\% CI=2.8-89.3); job opportunities (OR=11.0;
95\% CI=1.9-63.9); working conditions of doctors (OR=78.2; 95\% CI=8.6-713.3); financial conditions of doctors $(\mathrm{OR}=64.8 ; 95 \% \mathrm{CI}=7.2-581.5)$; and political conditions $(\mathrm{OR}=10.1 ; 95 \% \mathrm{CI}=1.1-94.1)$.

\section{Societal Expectations}

Eighty nine percent of respondents agreed that the Lebanese society expects them to train abroad. The respective percentages of students who thought that the following groups considered doctors trained abroad as more qualified were: the general public (84\%), patients (66\%), the academic community (59\%), and healthcare institutions (56\%). About half (48\%) the students considered doctors trained abroad as more qualified.

The association between theintention to train abroad and beliefs that the general public considers doctors trained abroad as more qualified than doctors trained in Lebanon wasstatistically significant(OR=6.3; 95\% CI=1.2-33.2). 


\section{Marketing of Abroad Training}

The conceptual framework related "marketing of abroad training" to getting information about abroad training from a number of sources (see Appendix, question 21). The percentages of students selecting those sources were as follows: the media (91\%), reports by medical students who completed elective rotations abroad (78\%), by comparing doctors trained locally to those trained abroad (66\%), reports by family members or friends living abroad (63\%), reports by doctors trained or training abroad (44\%), and own observations while on elective rotations abroad (16\%).

Theassociation between the intention to train abroad and receiving information about abroad training from doctors who trained or are training abroadwas statistically significant $(\mathrm{OR}=9.7 ; 95 \% \mathrm{CI}=1.7-56.6)$.

\section{Culture of Migration}

The conceptual framework defined a "culture of migration" in relation to a number of situations (see Appendix, questions 16-20). The percentages of students who reported being affected those situationswere as follows: residents traveling to retrain abroad (91\%); attendings encouraging students to train abroad (79\%); and having family members or friends living abroad and who would assist in traveling to train abroad (63\%). Only 33\% reported the presence of attendings who would assist them in traveling to train abroad while $24 \%$ reported having completed an elective rotation abroad. Half of respondents reported planning for elective rotations abroad with top destination countries being the US (64\%), France (12\%) and the United Kingdom (5\%).

The associations between the intention to train abroad and the 2 following factors were statistically significant: having family members or friends living abroad $(\mathrm{OR}=6.9 ; 95 \%$ $\mathrm{CI}=1.4-35.7)$ and witnessing residents traveling to retrain abroad (OR=22.2; 95\% CI=4.5-111.1).

\section{Barriers}

Respondents considered the following factors as barriers to train abroad: the expenses associated with training abroad (53\%), the required process of certification (52\%), and getting a visa (40\%).

Theassociations between the intention to train abroad and considering the 2 following factors as barriers were statistically significant: certification process $(\mathrm{OR}=6.5$; $95 \% \mathrm{CI}=1.1$ $38.7)$ and getting a visa $(\mathrm{OR}=12.7 ; 95 \% \mathrm{CI}=1.2-132.8)$.

\section{Modified Conceptual Framework}

The modified framework depicts the main influential factors found to be independently associated with the intention to migrate (Fig. 1). It also depicts the most prevalent additional factors and highlights (in bolded font) the ones that are independently associated with the intention to migrate.

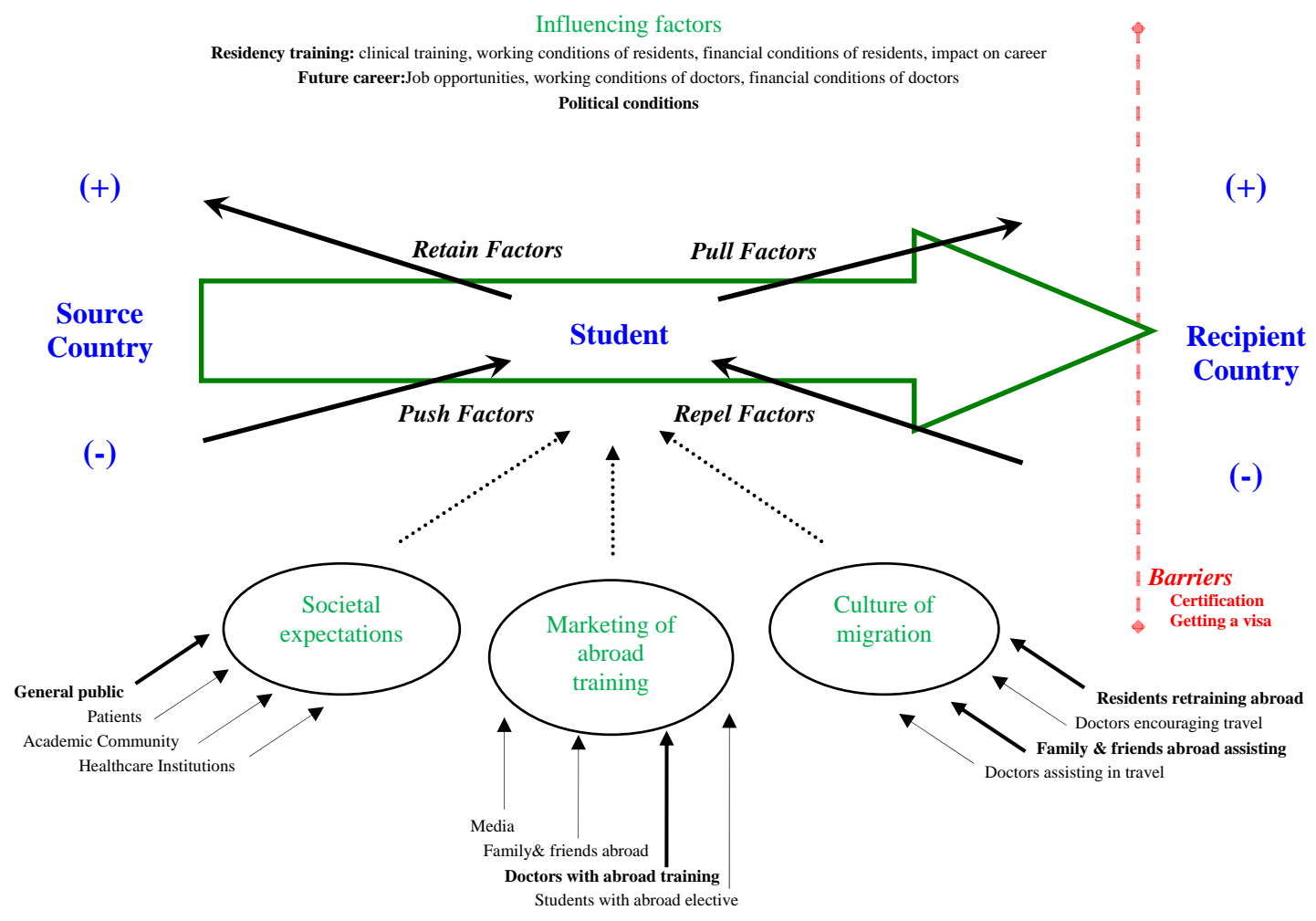

Bold arrows indicate factors independently associated with intention to migrate. Influential factors listed are those independently associated with intention to migrate.

Fig. (1). Modified conceptual framework for medical students' decisions to train abroad; survey study. 


\section{DISCUSSION}

In summary, the factors independently associated with the intention to train abroad relate to residency training, concern with impact on future career and political conditions. The following factors were also found to be important: thinking that the general public considers doctors trained abroad as more qualified than doctors trained in Lebanon; receiving information about abroad training from doctors who trained or are training abroad; having family members or friends living abroad; and feeling encouraged to train abroad by witnessing residents traveling to retrain abroad.

The modified conceptual framework illustrates the relationship between the factors explored in this study and the intention to migrate. It also expresses the relative strengths of these relationships with bold arrows for factors independently associated with intention to migrate. This makes the framework potentially modifiable to fit other specific contexts in which it might be explored in the future.

This study has a number of strengths. First, the validity of the framework in general and of the study questionnaire in particular is strengthened by the fact that it is based on the results of a qualitative study [11]. Second, the relatively high response rate decreases the likelihood of response biasand increases the validity of the results. Third, while we identified one conceptual model of factors influencing Ugandan nursing students' practice intentions [12], we were not able to identify any theoretical framework relating to the intentions of medical students to train abroad or to the international migration of physicians.

This study has some limitations. First, the evidence of an association between the framework factors and the intention to train abroad only suggests but does not prove causality. Second, the intention to train abroad may not translate into actual abroad training or eventual migration. However, this is likely to be the case given the extent and the trend of the migration of Lebanese medical graduates in the past [9] and the fact that abroad training represents a critical step in the migration of physicians [13]. Third, the high percentage of students expressing an intention to train abroad (96\%) probably reduced our power to show the significance of associations. It should have not however affected the validity of the results. Fourth, we were unable to distinguish whether a motivating factor was a push factor or a pull factor or both. Finally, it was possible that a student participated more than once given the survey was anonymous and confidential. However, we believe this was unlikely.

Civil instability was the chief motivator for the migration of Lebanese medical graduates in the 1970's [14]. Three decades later these political conditions are still a concern. Underlying fear of conflicts is a persisting phenomenon in Lebanon. Since we collected the study data in late 2005, factors related to civil and political instability may have again gained in importance due to recent political tensions at the national level and military conflicts at the regional level.

Factors related to residency training and future career (e.g. impact of residency training on future career, job opportunities, working conditions of doctors, and financial situation of doctors) were the other dominating factors in our study. Unfortunately we were not able to identify any study related to the quality of residency training in Lebanon to confirm students' dissatisfaction. Students' concern with future career is apparently related to the crisis of oversaturation of the Lebanese physician job market [15]. In fact, Lebanon has a physician density of 325 physician per 100,000 (2001 data), the second highest in the Middle East and North Africa [16]. As revealed by our earlier qualitative study, students travel abroad to gain a competitive advantage in the oversaturated job market [11].

It is counter-intuitive that although the certification process and obtaining a visa were perceived as barriers, they were associated with increased odds of intending to train abroad. This might be related to the fact that those who intend to train abroad are more aware of and concerned with such barriers.

Lebanon shares with developed countries affected by the international migration of physicians some of the factors motivating graduating medicalstudents to train abroad. In a recent survey of first-year house officers practicing in New Zealand, the $65 \%$ of respondents who intended to leave the country within 3 years of graduating were motivated by overseas travel, financial opportunities, job and training opportunities, and student loan debt [17]. In another survey of final year medicalstudents and junior doctors in New Zealand, $69 \%$ of respondents planned to work overseas, mostly to travel (70\%) and to further their professional training (58\%) [18]. In a 2004 survey of Croatian final year medical students, $33 \%$ of respondents would have considered emigration if they failed to get the desired specialty [19]. While the above specific motivators vary by country, they all fit the proposed theoretical framework.

The culture of migration and the marketing of abroad training seem to be prevalent in academic institutions raising major concerns. Similarly to our findings, investigators exploring migration of West African physicians described a "well-developed culture of medical migration” [20]. Professors and family members also acted as sources of information for West African students about the benefits of migration. These facts indirectly validate the proposed theoretical framework.

\section{CONCLUSION}

We plan to further validate the proposed conceptual framework by following up this cohort of students and comparing their future country of residency training and migration status with their reported intentions.There isalso a need to further validate the frameworkin other countries (especially those suffering from physician migration) and using actual migration as the outcome. The framework could serve as the basis for future research exploring factors affecting intentions of migrant physicians to return to their home countries [21].

Lebanese health policy makers need to take into account the findings of this study and develop strategies to address the problemof migrations of physicians [22-24], as well as other healthcare workers [25]. Such strategies should carefully address the oversaturation of the Lebanese job market and enhance residency training in terms of capacity, to make it responsiveness to the local market needs, and in terms of 
quality, to make it appealing for graduating medical students. Research, regulations and monitoring processes are needed to support these strategies.

\section{ACKNOWLEDGEMENTS}

We thank all medical students who participated in the survey. We thank Dr. Amy Hagopian for her valuable input. We also thank Dr. Bechara Chahoud, Dr. Abir Abdo, Dr. Stella Major, Dr. Claude Afif, Dr. Jacques Choucair, Dr. Mazen Sakr and Mme Hyam Kahi for their precious assis- tance. Holger Schünemann is supported by an EU Marie Curie Reintegration Grant (IGR 42192). This study was not funded. The authors declare that they have no competing interest in the area of study. The corresponding author had full access to all the data in the study and had final responsibility for the decision to submit for publication.

\section{CONFLICT OF INTEREST}

None declared.

\section{APPENDIX: SURVEY QUESTIONNAIRE}

\section{Student Characteristics}

1. Your age (Please circle the right answer)

$\begin{array}{ccccccccccc}<21 & 21 & 22 & 23 & 24 & 25 & 26 & 27 & 28 & 29 & 30 \\ 31 & 32 & 33 & 34 & 35 & 36 & 37 & 38 & 39 & 40 & >40\end{array}$

Gender

$\square$ 1. Female $\square$ 2. Male

Marital Status

$\square$ 1. Single $\square$ 2. Married $\square 3$. Divorced $\square$ 4. Widow

Religion:

\section{Lebanese Citizenship}

$\square$ 1. Yes $\square 2$. No. Citizenship:

Do you have a $2^{\text {nd }}$ citizenship or permanent residency?

$\square 1 . \quad$ No

Year of Medical School

$\square$ 1. Pre-final year

Your estimated overall ranking in class

$\square 1 . \quad$ Top $1 / 3$

$\square$ 2. Middle $1 / 3$

$\square 3 . \quad$ Bottom $1 / 3$

What type of residency training do you plan to do? (check only one)

$\square$ 1. None. I desire to be a general practitioner $\square 2$. Surgical

⒊ Medical

$\square$ 4. Other:

How would you rate your socio-economic status?

$\square$ 1. Lower $\square 2$. Lower middle $\square 3$. Upper middle $\square$ 4. Upper

Abroad training

Do you intend to do your training abroad?

$\square$ 1. No

$\square$ 2. Yes, for specialty training

$\square$ 3. Yes, for subspecialty training, after completing specialty training in Lebanon

If "yes" to q.10, what country would be your first choice? (check only one)

$\square$ 1. United Kingdom $\square$ 2. Canada

$\square 3 . \quad$ France

$\square$ 4. United States

$\square$ 5. Other:

If "yes" to q.10, what is your intention after you finish training abroad? (check only one)

$\square$ 1. Return directly to Lebanon

$\square$ 2. Work abroad for less than 5 years then return to Lebanon 
$\square$ 3. Work abroad for 5-10 years then return to Lebanon

$\square$ 4. Work abroad for more than 10 years then return to Lebanon

$\square$ 5. Never return to Lebanon

If "yes” to q.10, which is the most important goal for you to achieve through training abroad (check only one)

$\square$ 1. Excel professionally

$\square$ 2. Prosper financially

$\square$ 3. Establish myself quickly

$\square$ 4. Achieve long-term stability and security

$\square$ 5. Keep options open in terms of working in or outside Lebanon

$\square$ 6. Obtain the citizenship of the country of training

$\square 7$. Other:

What are the barriers for you to train abroad? (check all that apply)

$\square$ 1. Required process of certification (exams and tests)

$\square$ 2. Expenses (exams, applications, traveling)

$\square$ 3. Getting visas

$\square$ 4. Other:

\section{Influencing Factors}

Do you have family members or friends living abroad who would assist you if you travel to train abroad?
$\square$ 1. Yes
$\square$ 2. No

Are there doctors in your university that encourage you to train abroad?

$\square$ 1. Yes $\square 2$. No

Are there doctors in your university that would assist you to travel and train abroad?

$\square$ 1. Yes $\square 2$. No

Does witnessing residents traveling to retrain abroad motivate you to train abroad yourself?

$\square$ 1. Yes $\square 2$. No

Have you completed an observership or an elective rotation abroad?

$\square$ 1. Yes, country:

$\square$ 2. No, but I am planning to, Country:

$\square$ 3. No, and I am not planning to

From where did you get your information about training abroad? (check all that apply)

$\square$ 1. Media (movies, TV series)

$\square$ 2. Reports by family members or friends living abroad

$\square$ 3. Reports by medical students who completed observership or elective abroad

$\square$ 4. Reports by doctors who trained or are training abroad

$\square$ 5. By comparing doctors trained locally to those trained abroad

$\square$ 6. My own observations while on observership or elective abroad

$\square 7$. Other:

Do you agree that the Lebanese society expects you to train abroad? (circle one number)

\begin{tabular}{llllll}
-3 & -2 & -1 & 0 & +1 & +2 \\
\hline I strongly disagree & & I neither agree or disagree & +3 \\
\hline
\end{tabular}


Which of the following groups in the population consider doctors trained abroad as more qualified than doctors trained in Lebanon? (check all that apply)
$\square$ 1. General public
2. Patients
प3. Academic community
$\square$ 4. Healthcare institutions
$\square$ 5. I personally consider so
6. None of the above

Rate the impact of each of the factors listed below on your motivation to train abroad using the following scale:

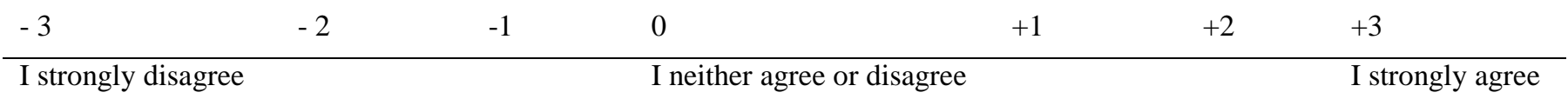

\section{Factors related to residency training}

Residency training opportunities

Availability of desired specialty, intensity \& fairness of competition

\section{Clinical training}

Exposure to cases, learning procedures, autonomy, application of theoretical learning

\section{Research training}

Financial resources, mentorship, possibility to publish

\section{Teaching in residency programs}

Doctors' and programs' commitment to teaching, presence of a curriculum

\section{Working conditions of residents}

Amount of work, relationship with doctors and nurses, rewarding and evaluation systems

\section{Financial situation of residents}

Income, financial independency, ability to start a family

\section{Impact of residency training on future career}

Ability to enter job market in Lebanon or abroad, chance of an academic career

\section{Factors related to working as a doctor}

\section{Job opportunities}

Intensity and fairness of competition, academic career opportunity, ability to work in your specialty of training

\section{Working conditions of doctors}

Amount of work, professional standards, advancement, continuous medical education

\section{Financial situation of doctors}

Income, stability of income

\section{Other factors}

\section{Personal conditions}

Issues related to partner, parents, children

\section{Social conditions}

$-3-2-10+1+2+3$

Social norms, social system, social relationships, social and family support, lifestyle, living dependently or independently

\section{Political conditions}

$-3-2-10+1+2+3$

Political situation, political system, ability to make changes, personal security

$$
\begin{aligned}
& -3-2-10+1+2+3 \\
& -3-2-10+1+2+3
\end{aligned}
$$$$
-3-2-10+1+2+3
$$$$
-3-2-10+1+2+3
$$$$
-3-2-10+1+2+3
$$

$-3-2-10+1+2+3$

$-3-2-10+1+2+3$

$-3-2-10+1+2+3$

$$
-3-2-10+1+2+3
$$

$-3-2-10+1+2+3$

Any comments? 


\section{REFERENCES}

[1] Gupta N, Diallo K, Zurn P, Dal Poz M. Assessing human resources for health: what can be learned from labour force surveys? Hum Resour Health 2003; 1: 5

[2] Forcier M, Simoens S, Giuffrida A. Impact, regulation and health policy implications of physician migration in OECD countries. Hum Resour Health 2004; 2: 12

[3] Akl EA, Mustafa R, Bdair F, Schünemann HJ. The US physician workforce and international medical graduates: Trends and characteristics. J Gen Intern Med 2007; 22: 264-8

[4] Chan B. Physician workforce planning: what have we learned? Lessons for planning medical school capacity and IMG policies. The Canadian perspective. Proceedings of the 7th International Medical Workforce Conference; 2003 September 11-14; Oxford, UK 2003.

[5] Cooper RA. Weighing the evidence for expanding physician supply. Ann Intern Med 2004; 141: 705-14.

[6] Gavel P. Physician workforce planning: What have we learned: an Australian perspective. Proceedings of the 7th International Medical Workforce Conference; 2003 Sep. 11-14; Oxford, UK 2003.

[7] Wanless D. Securing our future health: taking a long-term view. Interim Report. London, HM Treasury 2002.

[8] Mullan F. The metrics of the physician brain drain. N Engl J Med 2005; 353(17): 1810-8.

[9] Akl EA, Maroun N, Major S, Chahoud B, Schünemann HJ. Graduates of Lebanese medical schools in the United States: an observational study of international migration of physicians. BMC Health Serv Res 2007; 7: 49.

[10] Akl EA, Maroun N, Major S, et al. Post-graduation migration intentions of students of Lebanese medical schools: a survey study. BMC Public Health 2008; 8: 191.

[11] Akl EA, Maroun N, Major S, et al. Why are you draining your brain: factors underlying decisions of graduating Lebanese medical students to migrate. Soc Sci Med 2007; 64: 1278-84.

[12] Nguyen L, Ropers S, Nderitu E, Zuyderduin A, Luboga S, Hagopian A. Intent to migrate among nursing students in Uganda: measures of the brain drain in the next generation of health professionals. Hum Resour Health 2008; 6:5.
[13] Gupta I, Goldar B, Mitra A. The case of India. In: Zarrilli S, Kinnon C, Eds. International trade in health services: a development perspective. Geneva: UNCTAD and WHO Joint Publications 1998.

[14] Kronfol NM, Sibai AM, Rafeh N. The impact of civil disturbances on the migration of physicians: the case of Lebanon. Med Care 1992; 30(3): 208-15.

[15] Kassak K, Ghomrawi H, Osseiran A, Kobeissi H. The providers of health services. In Lebanon: a survey of physicians. Hum Resour Health 2006; 4: 4.

[16] Global Health Atlas. World Health Organization 2005. Available from: http://globalatlas.who.int/globalatlas/default.asp

[17] Moore J, Gale J, Dew K, Simmers D. Student debt amongst junior doctors in New Zealand; part 2: effects on intentions and workforce. N Z Med J 2006; 119: U1854.

[18] Zarkovic A, Child S, Naden G. Career choices of New Zealand junior doctors N Z Med J 2006; 119(1229): U1851.

[19] Kolcic I, Polasek O, Mihalj $\mathrm{H}$, et al. Research involvement, specialty choice, and emigration preferences of final year medical students in croatia. Croat Med J 2005; 46: 88-95.

[20] Hagopian A, Ofosu A, Fatusi A, et al. The flight of physicians from West Africa: views of African physicians and implications for policy. Soc Sci Med 2005; 61: 1750-60.

[21] Badr KF, Akl EA. Regionalizing healthcare: a vision for transforming Lebanon into a regional academic hub. BMC Health Serv Res 2010; 10: 167.

[22] Ahmad O. Managing medical migration from poor countries. $\mathrm{Br}$ Med J 2005; 331: 43-5.

[23] Labonte R, Packer C, Klassen N. Managing health professional migration from sub-Saharan Africa to Canada: a stakeholder inquiry into policy options. Hum Resour Health 2006; 4: 22.

[24] Dal Poz M, Quain E, O'Neil M, McCaffery J, Elzinga G, Martineau T. Addressing the health workforce crisis: towards a common approach. Hum Resour Health 2006; 4: 21.

[25] El-Jardali F, Alameddine M, Dumit N, Dimassi H, Jamal D, Maalouf S. Nurses' work environment and intent to leave in Lebanese hospitals: Implications for policy and practice. Int J Nurs Stud 2011; 48: 204-14.

(C) Akl et al.; Licensee Bentham Open.

This is an open access article licensed under the terms of the Creative Commons Attribution Non-Commercial License (http://creativecommons.org/licenses/by$\mathrm{nc} / 3.0 /$ ), which permits unrestricted, non-commercial use, distribution and reproduction in any medium, provided the work is properly cited. 